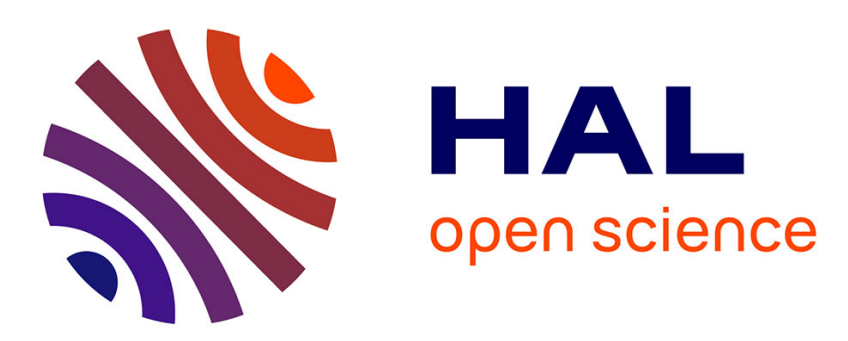

\title{
Chromatic correlations at injection and related ejection problems in separated sector cyclotrons
}

\author{
A. Chabert, J. Fermé, G. Gendreau, P. Lapostolle, P. Yvon
}

\section{To cite this version:}

A. Chabert, J. Fermé, G. Gendreau, P. Lapostolle, P. Yvon. Chromatic correlations at injection and related ejection problems in separated sector cyclotrons. 8th Particle Accelerator Conference, Oct 1979, San Francisco, United States. pp.3612-3614. in2p3-00183781

\section{HAL Id: in2p3-00183781 https://hal.in2p3.fr/in2p3-00183781}

Submitted on 30 Oct 2007

HAL is a multi-disciplinary open access archive for the deposit and dissemination of scientific research documents, whether they are published or not. The documents may come from teaching and research institutions in France or abroad, or from public or private research centers.
L'archive ouverte pluridisciplinaire $\mathbf{H A L}$, est destinée au dépôt et à la diffusion de documents scientifiques de niveau recherche, publiés ou non, émanant des établissements d'enseignement et de recherche français ou étrangers, des laboratoires publics ou privés. 
(C) 1979 IEEE. Personal use of this material is permitted. However, permission to reprint/republish this material for advertising or promotional purposes or for creating new collective works for resale or redistribution to servers

or lists, or to reuse any copyrighted component of this work in other works must be obtained from the IEEE.

IEEE Transactions on Nuclear Science, Vol. NS-26, No. 3, June 1979

CHROMATIC CORRELATIONS AT INJECTION AND RELATED EJECTION

PROBLEMS IN SEPARATED SECTOR CYCLOTRONS

by A. CHABERT, J. FERME, G. GENDREAU, P. LAPOSTOLLE and P. YVON.

GANIL (Grand Accélérateur National d'Ions Lourds) CAEN, France

\section{Abstract}

Injection into a cyclotron, in order to prevent emittance and phase spread dilution, requires proper couplings in the matching. One must first introduce a $\Delta \varphi / r^{\prime}$ associated with an $r / \Delta W$ coupling (through simplectic conditions); according to the angle of the accelerating dees and the choice of harmonic number the $\left(r, r^{\prime}\right)$ acceptance may also have to be tilted. All these effects are investigated in the case of the GANIL SSC's. At extraction corresponding correlations exist. For a resonant system, extraction may be difficult when the energy spread is large because of the large coupling induced by resonance. A precessional extraction which has been studied might in this case be more efficient. Other ways for making extraction easier are also considered. Moreover a new method of phase compression at injection into the SSC is presently under study at GANIL.

\section{Introduction}

GANIL is a complex cyclotron system made of two SSC's in cascade following a small compact injector cyclotron. Its purpose is to accelerate all ions from an energy of $10 \mathrm{MeV} / \mathrm{A}$ for very heavy ions up to $100 \mathrm{MeV} / \mathrm{A}$ for light ones. Emphasis is put on beam quality to be delivered from that installation and to the intensity produced.

Maximizing intensity leads to accept as much emittance and phase spread as possible; furthermore because flat-topping will not be available, at least at the beginning of operation, extreme care must be taken not to let these emittance and phase spread increase above the intrinsic limits. In order to do so, matching problems have been devoted a particular attention at injection into the SSC's but extraction also deserves consideration.

\section{Matching into a cyclotron [A1]:[A2]}

In a SSC, there is in general no problem for the axial motion but there exists an intrinsic correlation between radial coordinate $r$ and energy deviation $\Delta W / W$ inside a bunch. It then seems good to introduce such a correlation at injection rather than injecting achromatically. Our first order approximate analysis will show that, if this is true, it is rather through the phase versus angle correlation associated from simplectic conditions.

2.1 Let us consider first the case of a classical cyclotron with a uniform magnetic field, and no acceleration. We use polar coordinates $R, \theta$ and define particles with respect to the center of a bunch by $r$ and 1 or $\varphi$ :

$$
1=-R_{0} . \varphi / h
$$

Ro being the orbit radius, $h$ the harmonic number and $\varphi$ the R.F. phase with respect to the center.

To first order, the motion in a bunch of particles of a given energy is obtained from equations:

$$
d r=(1-10) \cdot d \theta ; d l=-r d \theta
$$

In the space $(1, r, d r / d \theta)$ a particle describes an ellipse in an oblique plane (fig. 1). Should a beam be injected in an ellipsoid with principal axis along 1 , $r, d r / d \theta$ coordinates it would start oscillating around $r$ axis and get a larger average phase length.
A correct matching requires a distribution possessing the $d r / d \theta=1$ plane of symmetry, that means, from equations (1), (2) and with $r^{\prime}=d r / d s$, the $\left(r^{\prime}, \boldsymbol{\varphi}\right)$ correlation :

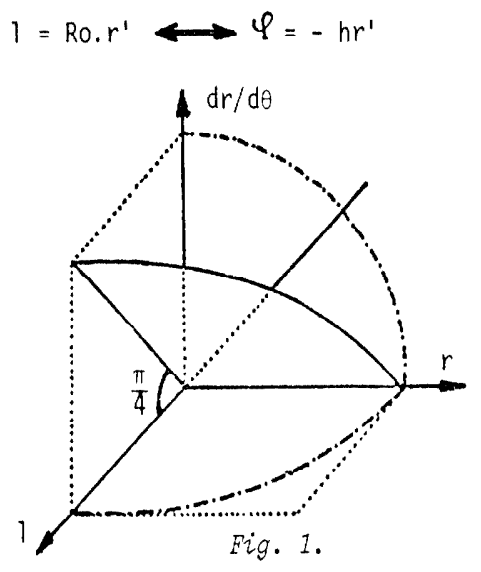

This correlation must be produced through the transfer system from an external achromatic section to some point inside the cyclotron. The general form of the transfer is given by:

$$
\left|\begin{array}{c}
r \\
r^{\prime} \\
1 \\
\Delta p / p
\end{array}\right|=\left|\begin{array}{llll}
a_{11} & a_{12} & 0 & a_{14} \\
a_{21} & a_{22} & 0 & a_{24} \\
a_{31} & a_{32} & 1 & a_{34} \\
0 & 0 & 0 & 1
\end{array}\right| \times\left|\begin{array}{c}
r \\
r^{\prime} \\
1 \\
\Delta p / p
\end{array}\right| 1
$$

Correlation (3) is obviously satisfied if

$$
a_{31}=\text { Ro. } a_{21} ; \quad a_{32}=\text { Ro. } a_{22}
$$

and, from simplectic conditions this solution appears to be the single one and entails:

$$
a_{14}=\text { Ro } \quad ; \quad a_{24}=0
$$

wherefrom comes the associated correlation :

$$
r=R o . \Delta W / 2 W_{0}
$$

Then, for injecting into a cyclotron, besides matching in $\left(r, r^{\prime}\right)$ and possibly in $\left(1, \Delta W / W_{0}\right)$ spaces, one must introduce coupling (3) or (7), the other one following automatically in practice, due to simplectic conditions.

2.2 In a separated sector cyclotron, the same properties exist. Looking in the center of a valley for instance, one now has :

$$
1=\text { Rvalley } \cdot r^{\prime} \leftrightarrow \varphi=-h r^{\prime} \cdot\left(R_{\text {valley }} / \bar{R}\right)
$$

and, provided $v_{r}$ is close enough to 1 , between two successive turns (putting $10=0$ ) :

$$
\begin{aligned}
& \Delta r=1 \cdot(\beta / R) \text { valley. } \Delta \theta ; \Delta \theta=2 \pi\left(\nu_{r}-1\right) \\
& \Delta 1=-r \cdot(R / B) \text { valley } \Delta \theta ; \Delta \theta
\end{aligned}
$$

The previous discussion still holds apart from a slight scaling in $r, r^{\prime}$ and 1 .

Curves 1 and 2 on figure 2 illustrate the influence of correlations (3) and (7) in the case of the separated four sectors cyclotron "CSS1" of GANIL. We can see the great influence of the $\left(r^{\prime}, \varphi\right)$ correlation on phase width pattern and associated energy spread; in this case of large $h$, the influence of the $(\Delta W / W, r)$ correlation on betatron oscillations, is much smaller. 
Note that the oscillations of $\Delta r$ and $\Delta \varphi$ have double frequency when the correlations are introduced. Remaining oscillations are due to acceleration, the effect of which will be now investigated.
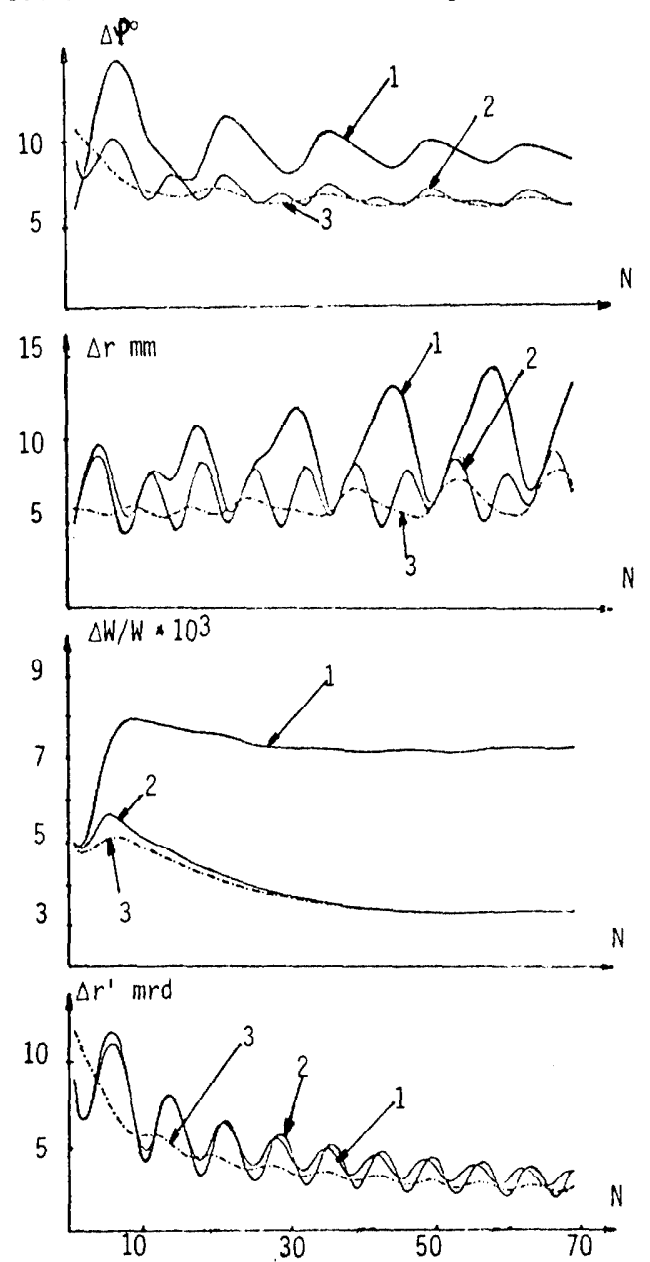

Fig. 2 Bunch widths $\Delta \varphi, \Delta r, \Delta W / W, \Delta r^{\prime}$ sompled at azimuth of injection (midale of a free valley) are defined as two times the standard deviation of the related distribution. Acceleration is similated in the GANIL CSS1 on harmonic 14.

Curves 1 : beam injected without any correlation $\Delta \varphi \varphi=6^{\circ}$, $\left.\Delta W / W=510^{-3}, E_{r}=45 \pi . m m . m r d=(5 \mathrm{~mm} \times 9 \mathrm{mrd}) . \pi\right)$. Curves 2 : same beam injected with $\left(r=R_{i n j} . \Delta W / W\right)$ and related $\left(i=\right.$ Rinj. $\left.r^{\prime}\right)$ correlations.

Curves 3 : same beam injected with an additional $\left(r, r^{\prime}\right)$ correzation (see par 3).

\section{Effects of acceleration}

For multi-dee accelerating systems it has been already mentioned that a bad choice of the dee angle may lead to difficulties [1], [2]. When crossing a dee of angle $2 . \delta$ a particle radially displaced by $r$ gets an extra relative energy gain $(a)$.

$$
\delta W / W_{0}=2 \text { A.r } / R
$$

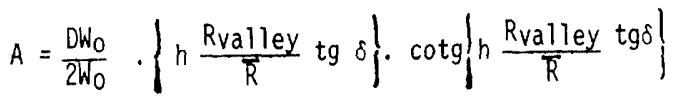

$D W_{0}$ being the energy gain of the non-displaced particle.

Let us consider a 4 SSC with two dees in opposite valleys and look at the orbits in a dee free valley.

(a) Note that the parameter A we introduce here corresponds to one dee and not to one turn.
Various matrix or geometric treatments can be used to compute the evolution of a particle along one turn. Let us just give an approximate description assuming $v_{r}=1$. Along one turn, an initial displacement 1 in the dee free valley is transformed in $r$ in the first dee ; according to (10) it only gives a displacement $r(1-A)$ after the first dee and $-r(1-A)^{2}$ after the second one with respect to the equilibrium orbit moving itself by $r A$ and $r A^{2}$ respectively after one and two dees.

Similarly an initial displacement $r$ gives - I at the first dee which, refering to (1) and adding the proper phase displacement and effect of radial kick (b) gives $r(1+A)$ after one half turn and $r(1+A)^{2}$ after one turn. Neglecting $A^{2}$ terms, one now has instead of $(9)$ :

$$
\begin{aligned}
& \Delta r=1 \cdot(B / R) \text { valley } \cdot \Delta \theta+2 \cdot A r \\
& \Delta 1=-r \cdot(R / B)_{\text {valley }} \cdot \Delta \theta-2 \cdot A 1
\end{aligned}
$$

Geometric relation (8) still holds and as said, there is during the turn only the very small extra energy gain

$$
\delta w / w_{0}=2 A^{2} .1 / \text { Rvalley }
$$

To our degree of approximation (12) can be replaced by:

$$
\begin{aligned}
\mathrm{d} \rho / \mathrm{d} \theta & =\lambda+k \rho ; \quad k=2 A / \Delta \theta=2 A / 2 \pi\left(v_{r}-1\right) \\
\mathrm{d} \lambda / \mathrm{d} \theta & =-\rho-k \lambda ; \\
\text { with } \quad \rho & =r \cdot \sqrt{(R / B)_{\text {valley }}} \\
\lambda & =1 \cdot \sqrt{(B / R)_{\text {valley }}}
\end{aligned}
$$

In the $(\rho, \lambda)$ plane-and correspondingly in the $(r$, ) or $\left(r, r^{\prime}\right)$ planes-the motion is no longer along a circle, but along the curve

$$
\rho^{2}+2 k \lambda \rho+\lambda^{2}=\text { const }
$$

and is obviousiy stable only if $|k|<1$. From (11), we see that $A$, that means $k$, decreases during acceleration and the ellipse comes closer and closer to the circle of figure 1. But, the difference may be quite important at injection where relative energy gain may be quite large and, in order to match the beam, besides correlations (3) and (7), the radial emittance should be elongated and tilted in the $\left(r, r^{\prime}\right)$ plane. Then, bunch breathing is reduced to a minimum in all coordinates.

We have considered here the beam in the middle of a free valley and of course different injection conditions would apply to other azimuths in the SSC.

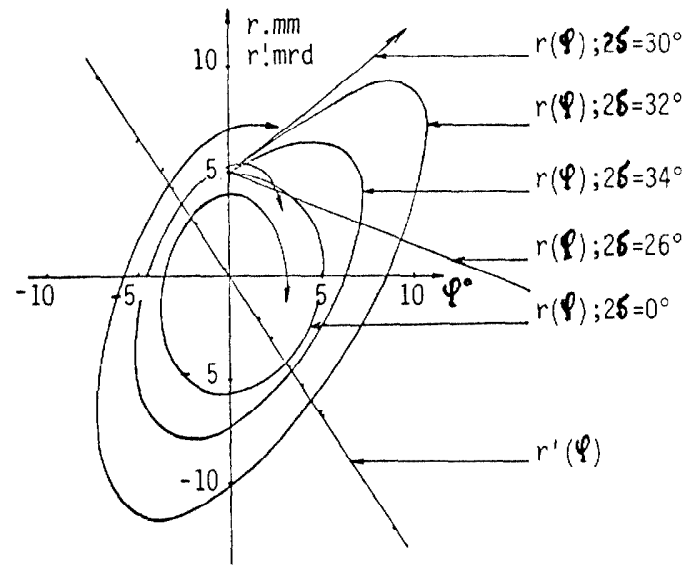

Fig. $3(r . \varphi)$ and $(r ! \varphi)$ patterms sampled at injection azimith of a particle in the GANIL CSST on harmonic $h=14$ showing the effect on acceleration of the dee angle

(GANIL cavity angle is $2 \times 17^{\circ}$ corresponding to ke.7.)

Curves of figure 3 illustrate the effects of acceleration in the GANIL "CSS1" when we change the dee

(b) The total phase displacement effect over one turm is zero when $r^{\prime}=0$ in the dees as seen in [1] but oniy in this case. 
angle that means the value of $k$. Curves 3 of figure 2 show the beam behaviour with all correlations established as well as possible; hopefully, beall behaviour remains good in a rather large range around the ideal values and correlations do not need to be established with a great accuracy.

\section{Extraction}

The internal beam presents before extraction the correlations described before ; then extraction and subsequent transport systems should take them into account in order to deliver achromatic beams.

When resonant extraction is used, it should be noticed that the coefficient of the $(\Delta W / W, r)$ and $\left(r^{\prime}, \varphi\right)$ correlations have been multiplied by the same factor the turn separation has been. This is one of the difficulties of this type of extraction where the contribution of energy spread to the beam width is increased in proportion of turn separation and only pure betatron contribution is not affected.

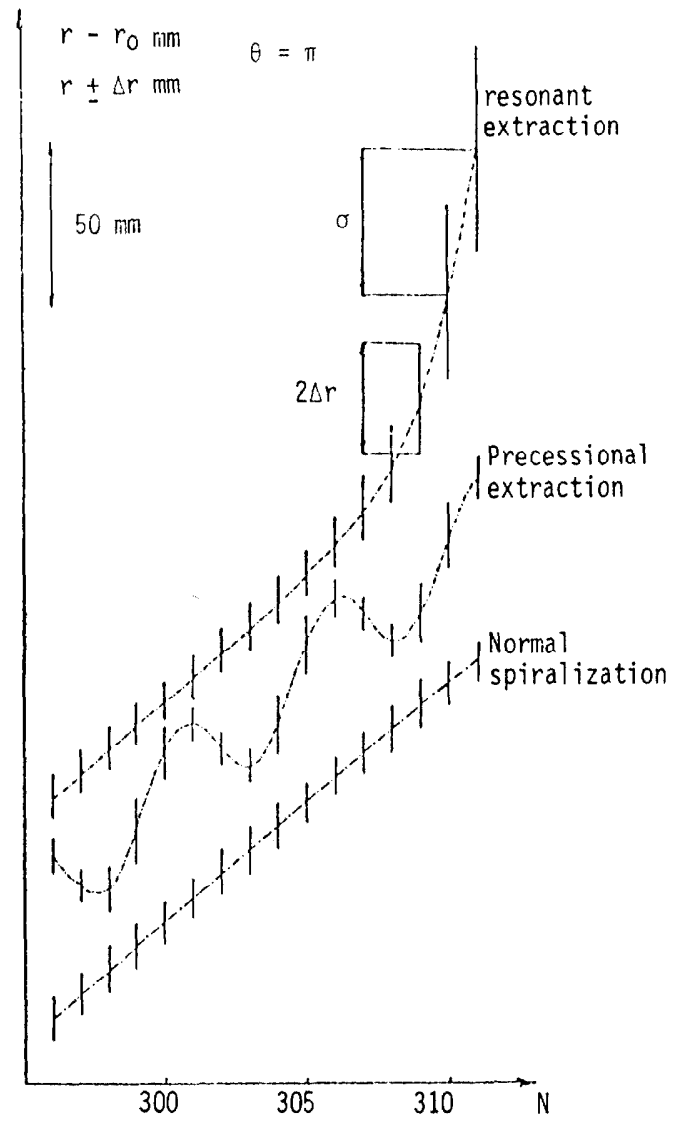

Fig. 4 Spiralization $\sigma$ and radial width $\Delta r$ near ejection in the GANIL "CSS2" ( $h=2$, Wejec $=100 \mathrm{MeV} / \mathrm{A})$. Energy dispersion of extracted becm has been choosen rather large $\left(\Delta \mathrm{W} / \mathrm{W}=2.10^{-3}\right)$ in order to show clearly the associated radial width increase in the case of resonant extraction. Precessional extraction-initiated in this case by a $\Delta V / V= \pm 30 \%$ on cavities- is much less sensitive to this large energy spread.

A precessional extraction making use of an oscillation established at injection can instead be considered. Schulte [3] has even shown that in this case energy spread contribution to beam width can be compensated for by a proper choice of $v_{r}$ at ejection. One may consider the following scheme for this type of extraction : orbit center position near ejection is function of turn $n$ through a relation of type :

$R N=A+\sigma N+a \cdot \cos \left(N \cdot \Delta \theta+\theta_{0}\right)$ $\sigma$ being the nominal turn separation, $a$ and $\theta_{0}$ the amplitude and phase of the precession, $\Delta \theta=2 . \pi\left(\nu_{r}-1\right)$.

The initial amplitude a of oscillation in the case of the precessional extraction is induced by an orbit perturbation either magnetic (very small bump near injection), electric (for instance unequal voltage on the dees), or by a change in the injection conditions $\left(r, r^{\prime}\right.$ or $\left.W\right)$. Optimization possibly includes a shift of $v_{r}$ at ejection, but this last effect is often contridactory to the one necessary to reduce beam chromatic width according to Schulte [3] .

The beam behaviour is illustrated on figure 4 in the case of the GANIL "CSS2".

\section{Conclusion}

Proper matching at injection into an SSC including various coupling effects avoids phase space dilution, permits large acceptance and reduces energy spread to a strict minimum. These effects strongly depend on the harmonic number and on the relative energy gain, the larger $h$ and DWo/Wo, the larger the effects.

In the absence of flat-topping, the energy spread may still be quite large when bunches are not short enough and we have shown how to extract the beam in this case. However, small energy spread may be necessary for other reasons and anyway ease the extraction problems. If one does not accept to reduce intensity by cutting the bunches (for instance by probes inside the SSC [4] there are possibilities to compress the phase by a voltage ramp [5] at beginning of acceleration. Moreover, we have recently found at GANIL that a slight departure from the isochronism law by means of a small field gradient on the first few turns may produce a very significant reduction of phase spread. The possibility of using this encouraging result is presently under study in conjunction with all its implications on injection matching and ejection problems.

\section{Bibliography}

1. W.M. Schulte, H.L. Hagedorn, Accelerated particles in an A.V.F. cyclotron with a one or two dee system. Proc. of the 8 th Inter. Conf. on Cyclotrons and their applications, BLOOMINGTON, 1978, to be published in I.E.E.E. N.S.

2. A. Chabert, G. Gendreau, P. Lapostolle. Limited energy spread in an SSC. Proc. of the 8 th Inter. Conf. on Cyclotrons and their applications, BLOOMINGTON, 1978, to be published in I.E.E.E. N.S.

3. W.M. Schulte. A method to obtain single turn extraction for large phase width in cyclotrons. Proc. of the 8th Inter. Conf. on Cyclotrons and the ir applications, BLOOMINGTON, 1978, to be published in I.E.E.E. N.S.

4. P. Lapostolle, J. Sauret. Réduction de la largeur en phase des faisceaux dans les CSS à l'aide de fentes. GANIL Report 78R/104/TP/05.

5. R.W. Muller, R. Mahrt. Phase compression and phase dilatation in isochronous cyclotrons. Nucl. Instr. and Meth. 86 (1970) pp. 241. 244

A1. G. Hinderer. Remarks on beam matching before injection into a split pole cyclotron, 13 ECPM, Milan, 1976.

A2.W.M. Schulte. The theory of accelerated particles in AVF Cyclotrons. Thesis 1978. EINDHOVEN. 